\title{
Integrated ST Segment Elevation Scores and In-hospital Mortality in STEMI Patients Undergoing Primary PCI
}

\author{
Diana Opincariu, Monica Chițu, Nora Rat, Imre Benedek
}

University of Medicine and Pharmacy, Tîrgu Mureș, Romania

\section{ABSTRACT}

The objective of this study was to study the integrated score of ST-segment resolution (ISSTE) and in-hospital death in patients undergoing primary percutaneous intervention (pPCI) for ST-segment elevation myocardial infarction (STEMI). Material and Methods: This prospective study included 586 consecutive patients admitted with STEMI to the Cardiology Clinic of the County Emergency Clinical Hospital of Tîrgu Mureș, between January $1^{\text {st }}$, 2013 and December 31, 2014, who underwent pPCI in less than twelve hours after the onset of symptoms. Clinical and demographic data were analyzed in 539 (91.9\%) survivors (Group 1) and 47 (8.1\%) nonsurvivors (Group 2). The Integrated Score of ST elevation (ISSTE) was calculated by summing the amplitude of the ST segment elevation in all the 12 leads, before and at 2 hours after revascularization. Results: The ISSTE score calculated at baseline, immediately before the primary percutaneous coronary intervention, was significantly higher in Group 2 as compared to Group $1(13.9 \pm 1.2$ vs. $11.0 \pm 0.2, p=0.026)$. At the same time, the ISSTE score calculated at 2 hours after the coronary intervention was significantly higher for patients in Group 2 ( $7.36 \pm 1.12$ vs. $2.9 \pm 0.1, \mathrm{p}<0.0001)$. Analysis of the dynamics of the ISSTE score indicated that patients who survived presented a more expressed reduction in the ISSTE score following pPCI, as compared to those who subsequently died (73.5\% reduction in Group 1 compared to $47.2 \%$ reduction in Group $2, \mathrm{p}<0.0001$ ). In-hospital mortality was significantly higher in the group of patients with $>50 \%$ reduction in the ISSTE score. The in-hospital death rate was $5.4 \%$ in patients with $>50 \%$ reduction in the ISSTE score, compared to $19.4 \%$ for those who presented less than 50\% reduction in the ISSTE score following pPCI ( $p<0.0001)$. The rate of successful reperfusion rate, expressed by the reduction in ISSTE score, was $83.8 \%$ in Group 1, compared to $55.3 \%$ in Group 2 ( $p<0.0001$ ), indicating that the absence of an efficient reperfusion after PPCI is associated with a higher mortality in STEMI patients, and could be evaluated using regression of the ISSTE score, which proved to be directly associated with mortality. Conclusion: The ISSTE score is shown to be an effective ECG-derived marker of myocardial damage in STEMI patients. A high ISSTE score is associated with higher mortality, while a reduction in the ISSTE score after pPCI may indicate an efficient reperfusion and a decrease in mortality in the first days after infarction.

Keywords: ISSTE score, STEMI, mortality, ST segment resolution

\section{ARTICLE HISTORY}

Received: 10 May, 2016

Accepted: 11 August, 2016

\section{Monica Chițu}

CORRESPONDENCE

Str. Gheorghe Marinescu nr. 38 540139 Tîrgu Mureș, Romania Tel: +40 265215551

E-mail: iuliachitu@yahoo.com 


\section{INTRODUCTION}

Early reperfusion with primary percutaneous coronary intervention (pPCI) has substantially improved outcomes for ST-elevation myocardial infarction (STEMI) patients. However, despite the efficiency of pPCI, in-hospital mortality after acute myocardial infarction (AMI) remains high. ${ }^{1}$

Detecting patients with an elevated risk for further events such as re-infarction or death, is necessary to initiate appropriate therapy and to reduce the risk of adverse events.

Prognostic evaluation for STEMI is far from ideal. Therefore, an ongoing search for markers to complete the currently applied risk scores is essential. ST segment resolution after pPCI or thrombolysis is indicative for efficient reperfusion at the level of the myocardial tissue. Several studies demonstrated that in STEMI patients, reversal of ST segment elevation is a good predictor for the patency of an infarct-related artery (IRA), and is inversely correlated with subsequent death. ${ }^{2-4}$ On the other hand, the persistence of ST segment elevation is specific for either an obstructed IRA or a patent artery, but with no myocardial and microvascular reperfusion (no-reflow phenomenon)..$^{4}$

The objective of this study was to study the integrated score of ST segment resolution (ISSTE) as an indicator of ECG-based score and in-hospital death in patients undergoing pPCI for STEMI.

The null hypothesis is that there is no statistical difference between patients who die during hospitalization and those discharged alive, for integrated score of ST segment resolution.

\section{MATERIAL AND METHODS}

This prospective study included all consecutive patients admitted to the Cardiology Clinic of the County Emergency Clinical Hospital of Tîrgu Mureș between January $1^{\text {st }}$, 2013 and December 31, 2014, with ST segment elevation myocardial infarction who underwent pPCI in less than twelve hours after the onset of symptoms. This resulted in 586 patients being enrolled in the study.

The inclusion criteria were:

- typical chest pain lasting for more than 10 minutes, with the onset less than 12 hours before presentation;

- troponin elevation;

- ST segment elevation of more than $1 \mathrm{~mm}$ in two or more consecutive leads, with reciprocal ST segment depression in opposite leads;

- pPCI performed in less than 12 hours from the onset of symptoms.
The exclusion criteria were:

- patients presenting with left bundle branch block (LBBB), which precluded the correct assessment of ST elevation on the surface ECG;

- the lack of any ST segment elevation on the ECG.

All patients underwent angiographic evaluation and revascularization via pPCI.

The study protocol was approved by the ethics committee of the institution, and all patients gave written informed consent before being enrolled in the study. The study was conducted by the principles stipulated in the Declaration of Helsinki.

Demographic data, medical history, clinical presentation, Killip class, serum biomarkers and angiographic recordings were recorded and analyzed in 539 (91.9\%) survivors (Group 1) and 47 (8.1\%) nonsurvivors (Group 2).

Twelve-lead electrocardiograms (ECGs) were obtained at presentation, immediately after PPCI and two hours after revascularization. The Integrated Score of ST Elevation (ISSTE) was calculated by summing the amplitude of the ST segment elevation in all twelve leads, before and at two hours after revascularization.

\section{STUDY ENDPOINTS}

The primary endpoint of the study was the all-cause mortality during hospitalization. The duration of stay in the hospital for the study population was 8.3 days (maximum), with an average of 6.5 days. All-cause mortality was recorded during this period. Only deaths that occurred during the hospitalization were included in the analysis.

The secondary endpoint of the study was the rate of successful reperfusion following PPCI, as defined by a reduction of $>50 \%$ of the ISSTE at two hours after pPCI.

\section{STATISTICAL ANALYSIS}

The data was statistically analyzed using GraphPad Prism 6.o. Quantitative values were expressed as mean \pm standard deviation, and categorical variables as frequencies and percentage values.

Statistical comparisons between the two study groups were performed using the $\mathrm{Chi}^{2}$ test or Fisher's exact test when suitable, for qualitative data.

For continuous values, the two groups were compared using the two-tailed Student t-test or the two-tailed Mann-Whitney test when suitable.

The relative performance of each test was evaluated with a $95 \%$ confidence interval (CI). 
TABLE 1. Patient demographics

\begin{tabular}{lccc}
\hline & $\begin{array}{c}\text { Group 1 } \\
\text { Survivors }\end{array}$ & $\begin{array}{c}\text { Group 2 } \\
\text { Deceased }\end{array}$ & p value \\
\hline Age (mean, y) & $61.4 \pm 11.7$ & $70.5 \pm 10.7$ & $<0.0001$ \\
Gender (female) & $226(41.9 \%)$ & $19(40.42 \%)$ & 0.8411 \\
\hline
\end{tabular}

The level of significance was set at alpha $=0.05$, against which the $\mathrm{p}$ values of each test are compared.

\section{RESULTS}

The null hypothesis of the study was rejected, as there was a significant statistical difference between the study groups in relation to the stated parameter (ISSTE resolution).

\section{BASELINE AND CLINICAL CHARACTERISTICS}

The mean population age was significantly higher in the nonsurvivor group compared to survivors $(61.4 \pm 11.7$ years in Group 1 vs. $70.5 \pm 10.7$ years in Group 2, p <0.0001) (Table 1). There was no significant difference between the study groups in respect to gender $(\mathrm{p}=0.8)$, the presence of hypertension $(\mathrm{p}=0.9)$, smoking status $(\mathrm{p}=0.4)$, obesity $(\mathrm{p}=$ $0.1)$ and previous myocardial infarction $(\mathrm{p}=0.6)$. However, compared to Group 1, Group 2 presented a significantly higher incidence of diabetes ( $48.9 \%$ vs. $21.5 \%, \mathrm{p}<0.0001$ ), previous stroke (19.1\% vs. $6.1 \%, \mathrm{p}=0.0009)$, or left ventricular dysfunction ( $85.0 \%$ vs. $49.0 \%$, p <0.0001) (Table 2).

Interestingly, higher rates of hypercholesterolemia and hypertriglyceridemia were recorded in the survivor group (Table 2).
Serum biomarkers characterizing myocardial necrosis and renal function were significantly elevated in the survivor group compared to the nonsurvivors (creatinkinase $3,035 \pm 385 \mathrm{mg} / \mathrm{dl}$ vs. $2,103 \pm 89.6 \mathrm{mg} / \mathrm{dl}, \mathrm{p}$ $=0.006$, creatinine $1.4 \pm 0.8$ vs. $0.9 \pm 0.6, \mathrm{p}<0.0001)$ (Table 3).

The clinical status at presentation was significantly more altered in patients who died during hospitalization compared to the survivor group, as expressed by: (1) the significantly higher incidence of Killip III and IV class in Group 2 (14.8\% Killip III and 36.1\% Killip IV in Group 2, compared to $2.8 \%$ Killip III and 4.6\% Killip IV in Group 1, $\mathrm{p}<0.0001$ ), and (2) the lower values of mean systolic arterial pressure in Group $2(104.7 \pm 31.13 \mathrm{mmHg}$ vs. $129.6 \pm$ $23.93 \mathrm{mmHg}, \mathrm{p}<0.0001$ ), indicating haemodynamic compromise in this group (Table 4).

\section{ANGIOGRAPHIC ANALYSIS}

The most frequent infarct-related artery was the left anterior descending coronary artery (LAD) in both groups, the difference between the groups being not statistically significant.

At the same time, angiographic analysis indicated a significantly higher number of cases with multi-vessel coronary disease among deceased patients ( $46.8 \%$ vs. $26.2 \%$, $\mathrm{p}=0.003, \mathrm{OR}=2.47,95 \% \mathrm{CI}=1.3-4.5)$. Post-procedural angiographic analysis indicated a significantly higher incidence of TIMI 3 flow following PPCI in the survivor group, as compared to the nonsurvivors ( $92.5 \%$ vs. $80.8 \%$, $\mathrm{p}=0.0054, \mathrm{OR}=2.94,95 \% \mathrm{CI}=1.3-6.5$ ), indicating a superior rate of successful reperfusion in the survivor group (Table 5).

TABLE 2. Risk factors and comorbidities in the study population

\begin{tabular}{|c|c|c|c|c|}
\hline & $\begin{array}{c}\text { Group 1 } \\
\text { Survivors } \\
\mathrm{n}=539(91.9 \%)\end{array}$ & $\begin{array}{c}\text { Group } 2 \\
\text { Deceased } \\
\mathrm{n}=47(8.1 \%)\end{array}$ & OR $(95 \% \mathrm{CI})$ & $\mathrm{p}$ value \\
\hline Diabetes & $116(21.5 \%)$ & $23(48.9 \%)$ & $3.4(1.8-6.4)$ & $<0.0001$ \\
\hline Hypertension & $461(85.6 \%)$ & $40(85.1 \%)$ & $0.9(0.4-2.2)$ & 0.913 \\
\hline Smoking & $216(40.1 \%)$ & $16(34.0 \%)$ & $0.7(0.4-1.4)$ & 0.411 \\
\hline Obesity & $211(39.2 \%)$ & $13(27.6 \%)$ & $0.5(0.3-1.1)$ & 0.117 \\
\hline History of stroke or TIA & $33(6.1 \%)$ & $9(19.1 \%)$ & $3.6(1.6-8.1)$ & 0.0009 \\
\hline History of angina pectoris & $257(47.7 \%)$ & $23(48.9 \%)$ & $1.0(0.5-1.9)$ & 0.878 \\
\hline Previous MI & $48(8.9 \%)$ & $5(10.6 \%)$ & $1.2(0.4-3.2)$ & 0.694 \\
\hline Peripheral artery disease & $22(4.0 \%)$ & $6(12.7 \%)$ & $3.4(1.3-8.9)$ & 0.0075 \\
\hline Left ventricular failure & $264(49.0 \%)$ & $40(85.0 \%)$ & $5.9(2.6-13.4)$ & $<0.0001$ \\
\hline Hiper-cholesterolemia & $243(45.1 \%)$ & $8(17.0 \%)$ & $0.2(0.1-0.5)$ & 0.0002 \\
\hline Hiper-triglyceridemia & $111(20.6 \%)$ & $1(2.1 \%)$ & $0.08(0.01-0.6)$ & 0.002 \\
\hline
\end{tabular}


TABLE 3. Baseline clinical and biological characteristics of the study groups

\begin{tabular}{lccc}
\hline & Survivors & Deceased & p value \\
\hline Heart rate (bpm) & $80.7 \pm 46.9$ & $89.4 \pm 25.8$ & 0.001 \\
SBP $(\mathrm{mmHg})$ & $129.6 \pm 23.9$ & $104.7 \pm 31.1$ & $<0.0001$ \\
DBP $(\mathrm{mmHg})$ & $76.2 \pm 13.1$ & $64.3 \pm 19.6$ & $<0.0001$ \\
Troponin $(\mathrm{ng} / \mathrm{ml})$ & $3.7 \pm 1.05$ & $5.5 \pm 2.0$ & 0.4551 \\
CK $(\mathrm{mg} / \mathrm{dl})$ & $2,103 \pm 89.6$ & $3,035 \pm 385$ & 0.006 \\
LDH $(\mathrm{u} / \mathrm{l})$ & $824.9 \pm 37.9$ & $1,385 \pm 223.2$ & 0.0066 \\
ASAT $(\mathrm{u} / \mathrm{l})$ & $232.9 \pm 9.8$ & $253.5 \pm 33.6$ & 0.7135 \\
ALAT $(\mathrm{u} / \mathrm{l})$ & $87.7 \pm 13.4$ & $110.1 \pm 21.5$ & 0.0361 \\
Glycemia $(\mathrm{mg} / \mathrm{dl})$ & $138.9 \pm 52.2$ & $201.5 \pm 105.6$ & $<0.0001$ \\
Hemoglobin $(\mathrm{mg} / \mathrm{dl})$ & $15.1 \pm 8.1$ & $13.9 \pm 2.7$ & 0.089 \\
Hematocrit $(\%)$ & $42.0 \pm 16.4$ & $39.5 \pm 5.4$ & 0.051 \\
Creatinine $(\mathrm{mg} / \mathrm{dl})$ & $0.9 \pm 0.6$ & $1.4 \pm 0.8$ & $<0.0001$ \\
Urea $(\mathrm{mg} / \mathrm{dl})$ & $38.6 \pm 17.2$ & $70.1 \pm 57.9$ & $<0.0001$ \\
WBC count/ml & $11,420 \pm 7,101$ & $14,274 \pm 4,868$ & 0.0002 \\
Platelet count/ml & $225,754 \pm 62,403$ & $235,590 \pm 82,418$ & 0.7679 \\
\hline
\end{tabular}

TABLE 4. Killip class on admission

\begin{tabular}{lccc}
\hline Killip class & Survivors & Deceased & p value, OR, 95\% CI \\
\hline I & $366(68.2 \%)$ & $11(23.4 \%)$ & $\mathrm{p}<0.0001, \mathrm{OR}=0.14[0.07-0.28]$ \\
II & $132(24.5 \%)$ & $12(25.5 \%)$ & $\mathrm{p}=0.8791, \mathrm{OR}=1.05[0.53-2.09]$ \\
III & $15(2.7 \%)$ & $7(14.8 \%)$ & $\mathrm{p}<0.0001, \mathrm{OR}=6.10[2.35-15.83]$ \\
IV & $25(4.6 \%)$ & $17(36.1 \%)$ & $\mathrm{p}<0.0001, \mathrm{OR}=11.63[5.67-23.84]$ \\
\hline
\end{tabular}

TABLE 5. Angiographic analysis

\begin{tabular}{lccc}
\hline & Survivors & Deceased & $\mathrm{p}$ value, OR, 95\% CI \\
\hline LAD & $257(47.76 \%)$ & $28(55.3 \%)$ & $\mathrm{p}=0.1, \mathrm{OR}=1.6[0.87-2.95]$ \\
Three vessel disease & $141(26.20 \%)$ & $22(46.80 \%)$ & $\mathrm{p}=0.003, \mathrm{OR}=2.4[1.35-4.53]$ \\
TIMI 3 flow post pPCI & $498(92.56 \%)$ & $38(80.85 \%)$ & $\mathrm{p}=0.005, \mathrm{OR}=2.94[1.33-6.53]$ \\
\hline
\end{tabular}

TABLE 6. Angiographic analysis

\begin{tabular}{lccc}
\hline & Survivors & Deceased & p value \\
\hline ISSTE before pPCI $(\mathrm{mm})$ & $11.08 \pm 0.29$ & $13.96 \pm 1.25$ & $<0.0001$ \\
ISSTE after pPCI $(\mathrm{mm})$ & $2.93 \pm 0.16$ & $7.36 \pm 1.12$ & $<0.0001$ \\
$>50 \%$ decrease in ISSTE & $26(55.31 \%)$ & $451(83.82 \%)$ & $<0.0001$ \\
$<50 \%$ decrease in ISSTE & $21(44.68 \%)$ & $87(16.17 \%)$ & $<0.0001$ \\
\hline
\end{tabular}

\section{THE INTEGRATED SCORE OF ST SEGMENT ELEVATION}

The ISSTE score calculated at baseline, immediately before the primary percutaneous coronary intervention, was significantly higher in Group 2 compared to Group 1 (13.9 \pm 1.2 vs. $11.0 \pm 0.2, p=0.026)$. At the same time, the ISSTE score calculated at two hours after the coronary interven- tion was significantly higher for patients in Group 2 (7.36 \pm 1.12 vs. $2.9 \pm 0.1, \mathrm{p}<0.0001$ ) (Figure 1 ). Analysis of the dynamics of the ISSTE score indicated that patients who survived presented a greater reduction in the ISSTE score following pPCI, compared to those who subsequently died (73.5\% reduction in Group 1 compared to $47.2 \%$ reduction in Group 2, p <0.0001) (Table 6). 

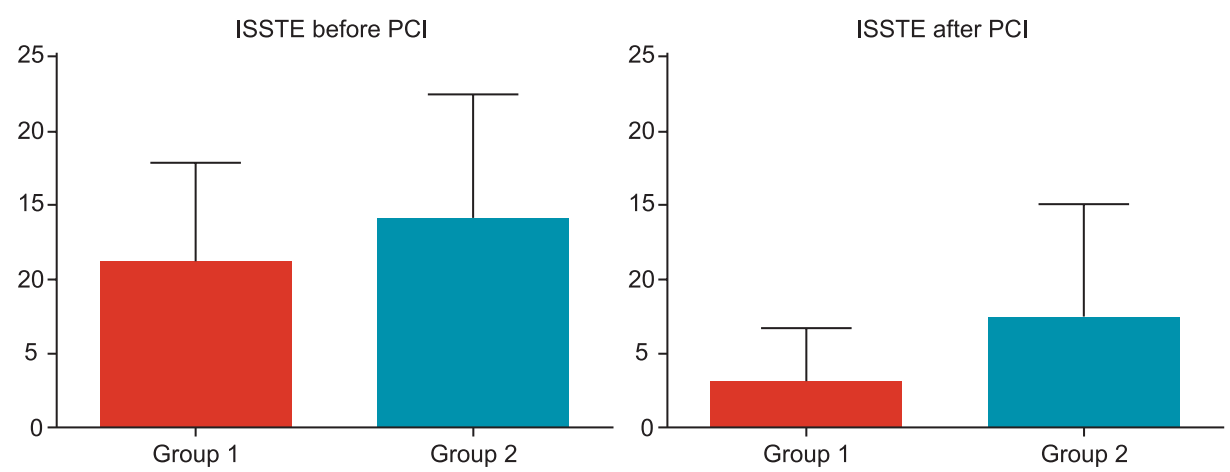

FIGURE 1. ISSTE score before and after revascularization (ISSTE in $\mathrm{mm}$ )

\section{STUDY ENDPOINTS}

\section{In-hospital mortality}

In-hospital mortality was significantly higher in the group of patients with $>50 \%$ reduction in the ISSTE score. The in-hospital death rate was $5.4 \%$ in patients with $>50 \%$ reduction in the ISSTE score, compared to $19.4 \%$ for those who presented less than $50 \%$ reduction in the ISSTE score following pPCI ( $\mathrm{p}<0.0001)$ (Figure 2$)$.

The mean duration of hospitalization was 6.4 days for those who survived, while in the group of patients who died, cardiac arrest occurred on average after 2.3 days following admission and pPCI.

\section{Reperfusion rate}

The rate of successful reperfusion, expressed by the reduction in the ISSTE score, was $83.8 \%$ in Group 1 compared to $55.3 \%$ in Group 2 ( $\mathrm{p}<0.0001$ ), indicating that the absence of an efficient reperfusion after PPCI is associated with a higher mortality in STEMI patients, and could be evaluated using regression of the ISSTE score.

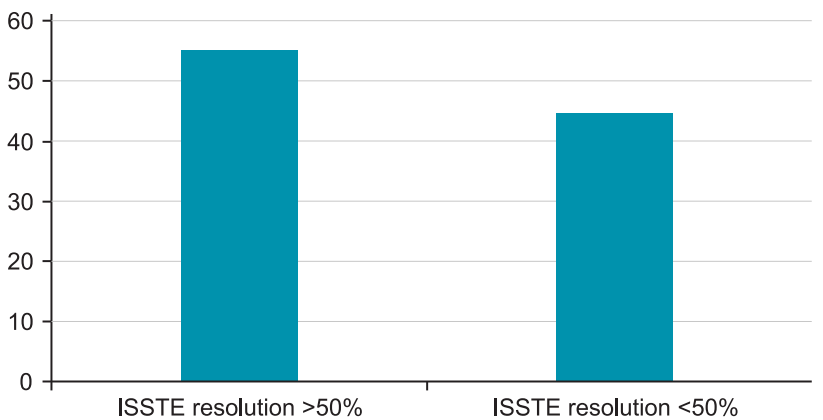

FIGURE 2. Mortality rates according to the ISSTE score decrease after $\mathrm{PCl}$

\section{DISCUSSIONS}

\section{STEMI MORTALITY IN THE STUDY POPULATION}

Widimsky et al. studied the reperfusion therapy for STEMI in 30 European countries, and found that the in-hospital mortality for pPCI-treated MI patients varied between $2.7 \%$ and $8 \%$. Similarly, in the present study population, $8.03 \%$ of patients died during hospitalization.

Early risk assessment is essential for STEMI patients. Therefore, there is a clear need to develop effective tools for assessing the risk of immediate fatal events in this patient population. This has generated the reevaluation of the prognostic usefulness of ST segment elevation decrease after STEMI.

\section{ECG SCORES FOR PREDICTING MORTALITY IN STEMI PATIENTS}

Previous studies have evaluated the predictive ability of early ST segment resolution after thrombolytic therapy. Schröder et al., in a substudy of the International Joint Efficacy Comparison of Thrombolytics (INJECT) trial, divided patients into three groups, based on ST segment resolution (STR) following thrombolytic administration. They defined the groups as (1) complete resolution ( $\geq 70$ STR), (2) partial resolution (70-30\%) and (3) no resolution $(<30 \%$ STR), and concluded that a complete STR $(\geq 70 \%)$ is associated with a lower mortality, while no STR $(<30 \%)$ predicts a very high early mortality. ${ }^{5}$ An analysis derived from Gruppo Italiano per lo Studio della Sopravvivenza nell'Infarto Miocardico (GISSI-2) trial, performed on 7,426 patients, evaluated the correlation between ST segment changes after fibrinolysis for myocardial infarction and the composed endpoint of in-hospital mortality plus congestive heart failure ( $\mathrm{CHF})$. In this study, the patients were categorized into two groups, the 
ECG cut-off for predicting coronary artery patency being $\geq 50 \%$ decrease in the ST segment elevation, after four hours of alteplase or streptokinase therapy. Their results showed that patients with $\geq 50 \%$ STR after thrombolytic administration, had a lower occurrence of the endpoint than patients with $<50 \%$ ST segment decrease $(16.2 \%$ vs. $22.9 \%$ respectively). ${ }^{6}$ Furthermore, in 2004, McLaughlin et al. evaluated the predictive power of various techniques for assessing ST segment resolution, after primary percutaneous coronary intervention performed for MI. Five methods were used to quantify STR, the thirty-day mortality rates being strongly associated with three of the five methods: $\Sigma$ STR (total \% STR across multiple leads), MaxSTR (\% STR in the lead with the highest ST elevation at baseline), MaxSTPost (absolute highest ST elevation after the intervention), and concluded that there was a strong correlation between mortality and ST segment resolution after pPCI. ${ }^{7}$

The present study proposes a method for measuring ST segment elevation, via mapping the ST segment elevation in all leads on the surface ECG. Based on this method, the Integrated Score of ST segment Elevation (ISSTE) is shown to be an effective tool that provides valuable prognostic information for post-MI early mortality, and allows easy stratification of patients into low- and high-risk groups. The ISSTE score was obtained by summing the amplitude of ST segment elevation in all leads, using the standard 12-lead ECG. ISSTE was calculated and then compared for both the pre- and two hours post-revascularization electrocardiograms. In line with previous studies, this method demonstrated that patients with $\geq 50 \%$ decrease in the ISSTE have a lower risk for mortality.

\section{CLINICAL PRESENTATION AND STEMI MORTALITY}

A study published in 2009 concluded that after pPCI, female gender is an independent predictor of death during hospitalization. ${ }^{8}$ However, in the current study, it was observed that there was a lower number of women in the deceased group (40.42\%) than in the survival group $(41.92 \%)$, though the difference was not statistically significant. An increased glycaemia can increase the mortality risk and negatively influence prognosis in post-MI patients. Capes et al. concluded in a meta-analysis that an acute increase in blood glucose concentrations is related to a higher risk of in-hospital death in both diabetic and nondiabetic patients. ${ }^{9}$ Also, Hsu PH et al. concluded, in a study comparing in-hospital outcome of STEMI patients with and without diabetes, that in-hospital mortality rate is higher for diabetic than nondiabetic patients. ${ }^{10}$
There was a larger number of diabetics among deceased patients $(48.93 \%)$, whereas only $21.56 \%$ of the survivors were diabetics $(\mathrm{p}<0.0001)$. The deceased patients also had higher blood glucose levels on admission when compared to survivors.

A study performed on 3,076 patients undergoing PCI for AMI, assessed the influence of the body mass index (BMI) on outcome, and concluded that BMI itself did not affect mortality during the hospital stay. ${ }^{11}$ However, another study, performed on 10,534 patients from the German MITRA PLUS Registry, showed that STEMI occur at a younger age in obese individuals, and that obesity cases showed, paradoxically, the lowest in-hospital mortality from all body mass index groups (obese $>30 \mathrm{~kg} / \mathrm{m}^{2}$, overweight $25-29.9 \mathrm{~kg} / \mathrm{m}^{2}$, average weight $18.5-24.9 \mathrm{~kg} /$ $\left.\mathrm{m}^{2}\right){ }^{12}$ Furthermore, a study that analyzed the in-hospital prognosis of percutaneous coronary interventions in extremely obese and normal weight patients, found that extreme obesity is an independent factor for assessing in-patient mortality. ${ }^{13}$ Das SR et al. showed, in a study on 50,149 patients, that morbidly obese patients suffering from STEMI are younger, and have less severe coronary atherosclerotic disease, better systolic function of the left ventricle and higher mortality rates compared to class I obese individuals. ${ }^{14}$

In the present study, there was a lower rate of obesity among the deceased (27.65\%) compared to survivors $(39.20 \%)$, although the difference was not significant ( $\mathrm{p}=$ 0.1179). Wang W et al. studied the correlation between cardiovascular risk factors and death rates in STEMI complicated with ventricular fibrillation, and one of their results revealed a strong correlation connecting smoking and mortality. ${ }^{15}$ The results of the present study showed that patients who died during hospitalization were less likely to have been active smokers.

A previous study, which aimed to determine the influence of hypertension on mortality rates after AMI, concluded that hypertension at the time of the acute coronary event is associated with higher in-hospital mortality. ${ }^{16}$ In the present study, we found an increased incidence of hypertension in the deceased group, compared to survivors.

In the present study, in the deceased group, there was a higher percentage of patients who had a history of angina pectoris ( $48.93 \%$ vs. $47.76 \%$ ), and a higher rate of patients who had previously suffered an MI (10.63\% vs. $8.92 \%$ ) when compared to the survivor group, in agreement with previously published results.

When analyzing the effects of prior stroke on in-hospital outcomes in MI patients, Abtahian et al. concluded that STEMI patients with previous stroke have an increased 
risk of death during hospitalization. ${ }^{17}$ In the current study cohort, when analyzing the deceased versus the survivor groups, $19.14 \%$ of patients who died had a positive history of stroke in comparison with survivors, where only $6.13 \%$ had a previous stroke $(\mathrm{p}=0.0009)$.

A study of early risk stratification in STEMI patients treated with pPCI based on leucocytes count, glycaemia and creatinine on admission showed that higher death rates were noted in cases with increased creatinine (cutoff value of $1.2 \mathrm{mg} / \mathrm{dl}) .{ }^{18}$ In the present study patients who died had higher levels of creatinine compared to patients who survived ( $1.4 \pm 0.8$ vs. $0.9 \pm 0.6, p<0.0001)$.

Lipšic et al. sought to find a relation between hemoglobin $(\mathrm{Hb})$ levels on admission and thirty-day mortality in MI. Their research, which included 1,841 patients admitted for MI, concluded that lower hemoglobin levels $(<10$ $\mathrm{g} / \mathrm{dl}$ ) on admission were associated with higher mortality rates. ${ }^{19}$ Although in the present study the mean hemoglobin on admission was not lower than $10 \mathrm{~g} / \mathrm{dl}$, the deceased patients had a lower $\mathrm{Hb}$ level $(13.97 \pm 2.774)$ compared to survivors $(15.11 \pm 8.165)(\mathrm{p}=0.089)$.

Shiraishi $\mathrm{J}$ et al. studied the predictive relevance of systolic blood pressure (SBP) on presentation, on the inhospital prognosis for STEMI patients who underwent pPCI. Their results showed that patients with an SBP of $141.158 \mathrm{mmHg}$ have better outcome, whereas an SBP $<105$ $\mathrm{mmHg}$ is associated with high death rates during hospitalization..$^{20}$ In our study, patients who deceased had lower SBP values on admission than survivors $(104.7 \mathrm{mmHg}$ vs. $129.6 \mathrm{mmHg}, \mathrm{p}<0.0001$ ). Furthermore, the deceased patients also recorded lower diastolic blood pressure values upon admission (64.38 mmHg vs. $76.27 \mathrm{mmHg}$, p $<0.0001$ ).

Another result of Shiraishi et al. was that Killip class $>$ III on presentation and multi-vessel coronary disease were independent factors in predicting the risk of death during hospital admission. ${ }^{20}$ In our results, from the total number of subjects that had deceased, $46.80 \%$ presented with three-vessel CAD, whereas, out of the survivors, only $26.20 \%$ were diagnosed with multivessel coronary disease. Regarding the Killip class of heart failure, we found that a higher percentage of patients who died had a Killip class IV score (36.17\%) compared to patients who survived (4.64\%). Killip class III was also better represented in the deceased group with $14.89 \%$ compared to $2.78 \%$ among patients who survived. Moreover, the majority (68.29\%) of the survivors were categorized as Killip class I, compared to only $23.40 \%$ in the deceased group.

\section{CONCLUSIONS}

The ISSTE score is a useful ECG-derived marker of myocardial damage in STEMI patients. A high ISSTE score is associated with higher mortality, while the regression of ISSTE after pPCI could indicate an adequate reperfusion and decreased mortality in the first days after infarction. Therefore, ISSTE could represent a simple, quick, inexpensive and effective method for categorizing patients into high and low risk of death during hospital stay following an acute myocardial infarction.

\section{CLINICAL IMPLICATIONS}

The Integrated Score of ST segment Elevation is a simple electrocardiographic tool, which can be used to stratify patients into low- and high-risk groups, according to the cut-off value of 50\% ST segment resolution after revascularization. Risk stratification is essential in the management of STEMI patients, as it optimizes patient triage for appropriate treatment, and improves monitoring during hospitalization and follow-up after discharge. Furthermore, the ISSTE should be used in addition to other risk scores that include clinical, laboratory and angiographic variables, in order to best predict in-hospital events for STEMI patients following revascularization.

\section{CONFLICT OF INTEREST}

None declared.

\section{ACKNOWLEDGEMENT}

This research was supported by the Cardio Med Medical Center, project no. CAM/2015/RD/17.

\section{REFERENCES}

1. Widimsky P, Wijns W, Fajadet J, et al. Reperfusion therapy for ST elevation myocardial infarction in Europe: description of the situation in 30 countries. Eur Heart J. 2010;31:943-957. doi: 10.1093/eurheartj/ehp492.

2. Schröder R. Prognostic Impact of Early ST segment Resolution in Acute Myocardial Infarction. Circulation. 2004;110:e506-e510. doi: 10.1161/01.CIR.0000147778.05979. E6.

3. McLaughlin MG, Stone GW, Amyong E, et al. Prognostic utility of comparative methods for assessment of ST segment resolution after primary angioplasty for acute myocardial infarction: the Controlled Abciximab and Device Investigation to Lower Late Angioplasty Complications (CADILLAC) trial. J Am Coll Cardiol. 2004;44:1215-1223. doi: 10.1016/j. jacc.2004.06.053. 
4. de Lemos JA, Braunwald E. ST segment Resolution as a Tool for Assessing the Efficacy of Reperfusion Therapy. J Am Coll Cardiol. 2001;38:1283-1294.

5. Schröder R, Weigscheider R, Schröder K, et al. Extent of early ST segment elevation resolution: a strong predictor of outcome in patients with acute myocardial infarction and a sensitive measure to compare thrombolytic regimens. A substudy of the International Joint Efficacy Comparison of Thrombolytics (INJECT) trial. J Am Coll Cardiol. 1995;26:1657-1664.

6. Mauri F, Maggioni AP, Franzosi MG, et al. A simple electrocardiographic predictor of the outcome of patients with acute myocardial infarction treated with a thrombolytic agent. A Gruppo Italiano per lo Studio della Sopravvivenza ell'Infarto Miocardico (GISSI-2) - Derived Analysis. J Am Coll Cardiol. 1994;24:600-607.

7. McLaughlin MG, Stone GW, Amyong E, et al. Prognostic Utility of Comparative Methods for Assessment of ST segment Resolution After Primary Angioplasty for Acute Myocardial Infarction - The CADILLAC trial. J Am Coll Cardiol. 2004 Sep 15;44:1215-1223. doi: 10.1016/j.jacc.2004.06.053.

8. Bename H, Taffet M, Bataille S, et al. Female gender still an independent predictor of in-hospital mortality after STEMI in the era of primary PCI? Insights from the Greater Paris Area PCI Registry. EuroIntervention. 2011;6(9):1073-9. doi: 10.4244/EIJV6I9A187.

9. Capes SE, Hunt D, Malmberg K, Gerstein HC. Stress hyperglycemia and increased risk after myocardial infarction in patients without diabetes: A systematic overview. Lancet. 2000;355(9206):773-778. doi: 10.1016/S01406736(99)08415-9.

10. Hsu PH, Jou YL, Lin PL, et al. Comparison of In-hospital outcome of Acute ST. Acta Cardiol Sin. 2011;27:145-151.

11. Kosuge $\mathrm{M}$, Kimura $\mathrm{K}$, Kojima $\mathrm{S}$, et al. Impact of body mass index on in hospital outcome after percutaneous coronary intervention for ST segment elevation acute myocardial infarction. Circ J. 2008;72:521-525.

12. Wienbergen H. Gih AK, Juenger $\mathrm{C}$, et al. Impact of the body mass index on occurrence and outcome of acute ST-elevation myocardial infarction. Clinical Research in Cardiology. 2008;97:83-88. doi: 10.1007/s00392-007-0585-x.

13. Payvar SP, Kim S, Rao E, et al. In hospital outcomes of percutaneous coronary interventions in extremely obese and normal weight patients. J Am Coll Cardiol. 2013;62:692-696. doi: 10.1016/j.jacc.2013.05.058.

14. Das SR, Alexander KP, Chen AY, et al. Impact of body weight and extreme obesity on the presentation, treatment, and in-hospital outcomes of 50,149 patients with ST segment elevation myorcardial infarction, results from the NCDR (National Cardiovascular Registry). J Am Coll Cardiol. 2011;58:2642-50. doi: 10.1016/j.jacc.2011.09.030.

15. Wang W, Chen QF, Yin RX, et al. Clinical features, risk factors, and treatment experience: a review of 74 patients with ST segment elevation myocardial infarction complicated by ventricular fibrillation. J Emerg Med. 2014;47:729-735. doi: 10.1016/j.jemermed.2014.06.041.

16. Kang DG, Jeong $\mathrm{MH}$, Ahn $\mathrm{Y}$, et al. Clinical effects of hypertension on mortality of patients with acute myocardial infarction. J Korean Med Sci. 2009;24:800-806. doi: 10.3346/ jkms.2009.24.5.800.

17. Abtahian F, Olenchock B, Ou FS, et al. Effect of prior stroke on the use of evidence-based therapies and in-hospital outcomes in patients with myocardial infarction (from the NCDR ACTION GWTG registry). Am J Cardiol. 2011;107:1441-6. doi: 10.1016/j.amjcard.2011.01.020.

18. Przylusk J, Kalinczuk L, Kruk M, et al. Early risk stratification in patients treated with primary PCI for STEMI based on simultaneous assessment of admission WBC count and levels of glycemia and creatinine. Circulation. 2006;114(Suppl18)

19. Lipšic E, van der Horst ICC, Voors AA, et al. Hemoglobin levels and 30-day mortality in patients after myocardial infarction. Int J Cardiol. 2005;100:289-292.

20. Shiraishi J, Kohno Y, Sawada T, et al. Prognostic impact of systolic blood pressure at admission on in-hospital outcome after primary percutaneous coronary intervention for acute myocardial infarction. J Cardiol. 2012;60:139-144. doi: 10.1016/j.jjcc.2012.02.008. 